1995

\title{
Activity Coefficients of Adsorbed Mixtures
}

Orhan Talu

Cleveland State University

Jianmin Li

Cleveland State University

Alan L. Myers

University of Pennsylvania

Follow this and additional works at: https://engagedscholarship.csuohio.edu/encbe_facpub

Part of the Thermodynamics Commons

How does access to this work benefit you? Let us know!

Publisher's Statement

The final publication is available at Springer via http://dx.doi.org/10.1007/BF00704999

\section{Original Citation}

Talu, O., Li, J., , \& Myers, A. L. (1995). Activity coefficients of adsorbed mixtures. Adsorption, 1(2), 103 112. doi:10.1007/BF00704999

\section{Repository Citation}

Talu, Orhan; Li, Jianmin; and Myers, Alan L., "Activity Coefficients of Adsorbed Mixtures" (1995). Chemical \& Biomedical Engineering Faculty Publications. 82.

https://engagedscholarship.csuohio.edu/encbe_facpub/82

This Article is brought to you for free and open access by the Chemical \& Biomedical Engineering Department at EngagedScholarship@CSU. It has been accepted for inclusion in Chemical \& Biomedical Engineering Faculty Publications by an authorized administrator of EngagedScholarship@CSU. For more information, please contact library.es@csuohio.edu. 


\title{
Activity Coefficients of Adsorbed Mixtures
}

\author{
ORHAN TALU AND JIANMIN LI \\ Department of Chemical Engineering, Cleveland State University, Cleveland, Ohio 44115 \\ ALAN L. MYERS \\ Department of Chemical Engineering, University of Pennsylvania, Philadelphia, Pennsylvania 19104
}

\begin{abstract}
Experimental and simulated data for adsorption of gas mixtures on energetically heterogeneous surfaces like activated carbon and zeolites exhibit negative deviations from ideality. The deviations are large in some cases, with activity coefficients at infinite dilution equal to 0.1 or less. Similar molecules form ideal mixtures, but molecules of different size or polarity are nonideal. Equations for bulk liquid mixtures (Wilson, Margules, etc.) do not apply to isobars for adsorbed mixtures. A two-constant equation for activity coefficients as a function of composition and spreading pressure is in good agreement with theory, simulation, and experiment.
\end{abstract}

Keywords: adsorption, mixtures, activity coefficients, zeolites

\section{Introduction: Vapor-Liquid Equilibrium (VLE)}

The "gamma/phi" approach is widely used (Van Ness and Abbott, 1982) to analyze and correlate experimental VLE data as a function of temperature $(T)$, pressure $(P)$, and composition. In this paper, the gamma/phi approach is extended to vapor-adsorbed phase equilibrium (VAE).

The fundamental equation of VLE is the equality of the fugacities in the gas phase $\left(f_{i}^{G}\right)$ and the liquid phase $\left(f_{i}^{L}\right)$. For component no. $i$ :

$$
f_{i}^{G}\{T, P, \mathbf{y}\}=f_{i}^{L}\{T, P, \mathbf{x}\}
$$

$\mathbf{y}$ and $\mathbf{x}$ are the vectors of gas-phase and liquid-phase mole fractions, respectively. The \{\} notation represents functionality. The gamma/phi approach is to write Eq. (1) as:

$$
y_{i} P \phi_{i}\{T, P, \mathbf{y}\}=x_{i} f_{i}^{\mathrm{sat}}\{T, P\} \gamma_{i}\{T, P, \mathbf{x}\}
$$

$f_{i}^{\text {sat }}$ is the fugacity of pure $i$ saturated liquid at the pressure and temperature of the mixture, $\phi_{i}$ is the fugacity coefficient of $i$ in the vapor phase and $\gamma_{i}$ is the activity coefficient of $i$ in the liquid phase, $\gamma_{i}$ and $\phi_{i}$ are correction factors for deviations from ideal behavior; their usefulness stems from the following facts (Prausnitz, Lichtenthaler and Azevedo, 1986);

1. The limiting value of $\phi_{i}$ at low pressure is unity;

2. The value of $f_{i}^{\text {sat }}$ at low pressure is the vapor pressure of the pure liquid $P_{i}^{\text {sat; }}$;
3. $\gamma_{i}$ has a limit of unity as $x_{i} \rightarrow 1$;

4. $\gamma_{1}$ and $\gamma_{2}$ in a binary system are related by the Gibbs-Duhem equation;

5. Values of $\phi_{i}$ are calculated using gas-phase second virial coefficients at low to moderate pressure, and cubic equations-of-state at high pressure;

6. The system of thermodynamic excess functions (excess Gibbs free energy $g^{\mathrm{ex}}$, excess enthalpy $h^{\mathrm{ex}}$, etc.) provides a concise mathematical description of the dependence of activity coefficients upon the independent variables $(T, P, \mathbf{x})$.

7. The effect of $P$ on $\gamma_{i}$ is small enough to be ignored in many cases;

Experimental activity coefficients for liquid mixtures are routinely reported using the excess Gibbs free energy function:

$$
\frac{g^{\mathrm{ex}}}{R T}=\sum_{i} x_{i} \ln \gamma_{i}
$$

In general $g^{\operatorname{ex}}\{T, P, \mathbf{x}\}$, but for low-pressure measurements the effect of pressure is insignificant. For an ideal solution (Raoult's law), $\gamma_{i}=1$ and $g^{\mathrm{ex}}=0$. Solutions are classified as having positive or negative deviations from ideality depending upon the sign of $g^{\mathrm{ex}}$. In general positive deviations $\left(g^{\mathrm{ex}}>0\right)$ are associated with mixtures of molecules with different polarities or different structures (e.g. mixtures of aliphatic and aromatic hydrocarbons) while negative deviations are associated with weak chemical bonding of unlike molecules (e.g, chloroform and acetone). 
The determination of $\gamma_{i}$ from experimental VLE data and the calculation of VLE diagrams from $g^{\text {ex }}$ data are covered in thermodynamic textbooks (Smith and Van Ness, 1975). The purpose of the introduction is to summarize the gamma/phi approach to VLE in preparation for its extension to VAE. In the next section, the many similarities and major differences between VLE and VAE are summarized.

\section{Thermodynamic Equations for Vapor Adsorp- tion Equilibrium (VAE)}

For VAE (Talu and Zwiebel, 1986) the equivalent of Eq. (1) is:

$$
f_{i}^{G}\{T, P, \mathbf{y}\}=f_{i}^{A}\{T, \Pi, \mathbf{x}\}
$$

$f_{i}^{G}$ is the gas-phase fugacity and $f_{i}^{A}$ is the adsorbedphase fugacity. $\Pi$, which has the units of surface ten$\operatorname{sion}(\mathrm{N} / \mathrm{m})$, is the pressure of the adsorbed solution. For VLE the conditions of thermal and mechanical equilibrium are implicit in equal values of $T$ and $P$ in both phases. For VAE there is thermal equilibrium but mechanical equilibrium is not attained because there is no movable wall separating the adsorbed phase from the gas phase. In fact the adsorbed phase has no volume according to the Gibbs definition of adsorption. This extra degree of freedom $(\Pi)$ for adsorption equilibrium is the essential difference between VLE and VAE. According to the Gibbs phase rule, binary VLE has two degree of freedom and binary VAE has three degrees of freedom.

For VAE, the gamma/phi approach analogous to Eq. (2) is (Myers and Prausnitz, 1965):

$$
y_{i} P \phi_{i}\{T, P, \mathbf{y}\}=x_{i} f_{i}^{\circ}\{T, \Pi\} \gamma_{i}\{T, \Pi, \mathbf{x}\}
$$

$f_{i}^{\circ}$ is the fugacity of pure $i$ adsorbate at the temperature and spreading pressure of the mixture.

Remarkably, there is no experimental method for direct measurement of $\Pi$ in microporous adsorbents. Moreover, except for flat surfaces, $\Pi$ cannot be determined from molecular simulation by ensemble averages or fluctuations. The only way to determine $\Pi$ is by integration of the Gibbs adsorption isotherm:

$$
A d \Pi=R T \sum_{i} n_{i} d \ln f_{i} \quad \text { (const. } T \text { ) }
$$

$n_{i}$ is specific amount adsorbed (mol $/ \mathrm{kg}$ ) and $f_{i}=$ $P y_{i} \phi_{i}$ is the gas-phase fugacity. Equation (6) plays a central role in adsorption thermodynamics for:
1. Calculation of spreading pressure from changes in bulk-phase properties;

2. Conversion of a surface-phase equation of state relating spreading pressure $(\Pi)$, molar area $(a)$, and $T$ into an adsorption isotherm;

3. Assessment of thermodynamic consistency of experimental data for adsorption of mixtures by integration of Eq. (6) over any closed path.

For adsorption of a pure gas, Eq. (6) simplifies to:

$$
\left.\frac{A d \Pi}{R T}=n d \ln f \quad \text { (const. } T\right)
$$

Using the reference state $\Pi=0$ at $P=0$ :

$$
\frac{\Pi A}{R T}=\int_{0}^{f} \frac{n}{f} d f \quad \text { (const. } T \text { ) }
$$

Inversion of Eq. (8) provides the function $f_{i}^{\circ}\{T, \Pi\}$, the standard-state fugacity in Eq. (5). If the pressure is near- or sub-atmospheric, the fugacity $f$ in Eq. (8) may be replaced by the gas-phase pressure $P$ :

$$
\frac{\Pi A}{R T}=\int_{0}^{P} \frac{n}{P} d P \quad \text { (const. } T \text { ) }
$$

The limit at zero coverage of the integrand in Eq. (9) is given by L'Hospital's rule:

$$
\lim _{P \rightarrow 0} \frac{n}{P}=\lim _{P \rightarrow 0} \frac{d n}{d P}=H
$$

The limiting slope $H$, called Henry's constant, is non-zero and finite. Some theoretical equations for the adsorption isotherm (e.g. the Freundlich equation and the Dubinin-Radushkevich equation) predict an infinite value of $H$; these equations are unsuitable for the calculation of spreading pressure and other thermodynamic properties (Talu and Myers, 1988). Surface-phase properties at zero coverage are a function of the interaction of a single adsorbate molecule with the surface of the adsorbent. The limit of zero surface coverage serves as the reference state for the properties of adsorbed solutions, in the same way that the perfect-gas state serves as the reference state for the configurational properties of bulk fluids.

A major difference between VAE and VLE is the effect of pressure upon activity coefficients. For VLE measured at low pressure up to several atmospheres, pressure has a weak effect upon thermodynamic properties of the liquid phase. For VAE, spreading pressure has a strong effect upon thermodynamic properties of the adsorbed phase such as activity coefficients. At the limit of zero coverage the molecules are too far apart to 
interact and the activity coefficients are equal to unity: $\lim _{(\Pi \rightarrow 0)} \gamma_{i}=1$. The degree of nonideality at higher coverage depends upon differences in polarity, size, and structure of the adsorbate molecules and upon the structure of the solid.

Another difference between VAE and VLE is the standard state. For VLE, the standard state is the pure liquid at the temperature and pressure of the mixture. For low-pressure VLE measurements, the effect of pressure upon the standard-state fugacity is weak and is usually ignored. For VAE, the standard-state fugacity $f_{i}^{\circ}$ is a strong function of $\Pi$.

The excess area $a^{\text {ex }}$ of an adsorbed solution is analogous to the excess volume of a bulk solution:

$$
a^{\operatorname{ex}}\{T, \Pi, \mathbf{x}\}=a\{T, \Pi, \mathbf{x}\}-\sum_{i} x_{i} a_{i}^{\circ}\{T, \Pi\}
$$

Excess Gibbs free energy and excess area are related by:

$$
a^{\mathrm{ex}}=\left(\frac{\partial g^{\mathrm{ex}} / R T}{\partial \Pi}\right)_{T, \mathrm{x}}
$$

Since molar area $a=A / n$, it follows from Eq. (11) that the total amount adsorbed from a mixture $n=$ $\sum_{i} n_{i}$ is

$$
\frac{1}{n}=\sum_{i}\left(\frac{x_{i}}{n_{i}^{\circ}}\right)+\frac{a^{\mathrm{ex}}}{A}
$$

The set of Eqs. (4)-(13) provides the thermodynamic framework for isothermal VAE. Given the behavior of the pure adsorbates, multicomponent systems can be characterized by the functional form of $g^{\operatorname{ex}}\{T, \Pi, \mathbf{x}\}$.

\section{Theories of Mixed-Gas Adsorption}

The functionality of the excess properties for VAE are examined for two theories: (1) the two-dimensional approximation, and (2) partial exclusion of large molecules from small micropores.

\subsection{Two-Dimensional (2-d) Approximation}

The (2-d) approximation is that the surface of the adsorbent is flat and adsorbed molecules are trapped at the bottom of the gas-solid potential energy well, with their translational motion effectively restricted to twodimensions parallel to the surface. The 2-d approximation applies to sub-monolayer adsorption on planar surfaces for which the depth of the gas-solid potential energy well $\left(-U_{1 \mathrm{~S}} / k T\right) \approx 10$ or more.
Henry's constant $H$ in Eq. (10) is related to the adsorption second virial coefficient $B_{1 \mathrm{~S}}$ by:

$$
H=\frac{B_{1 \mathrm{~S}}}{R T}
$$

$B_{1 S}$ is a function of the gas-solid potential energy $U_{1 \mathrm{~S}}$ (Steele, 1974) of a single adsorbate molecule (1) with the surface $(\mathrm{S})$ :

$$
B_{1 \mathrm{~S}}=\int_{V}\left[e^{-U_{\mathrm{S}} / k T}-1\right] d V
$$

The integral is over the specific volume $V\left(\mathrm{~m}^{3} / \mathrm{kg}\right)$ accessible to the adsorbate molecule. If the temperature is high enough to ignore lateral variations in $U_{1 \mathrm{~S}}$ :

$$
B_{1 \mathrm{~S}}=A \int_{0}^{\infty}\left[e^{-U_{1 \mathrm{~S}} / k T}-1\right] d x
$$

where $A$ is the specific surface area $\left(\mathrm{m}^{2} / \mathrm{kg}\right)$ of the adsorbent and $x$ is the distance from the surface.

The 2-d compressibility factor $z$ is defined by (Van Ness 1969):

$$
z \equiv \frac{\Pi a}{R T}
$$

The thermodynamic equation for the calculation of fugacity in a 2-d adsorbed mixture from a spreading pressure-explicit equation of state is (Hoory and Prausnitz, 1967):

$$
f_{i}=\frac{n_{i} R T}{B_{i \mathrm{~S}}} \exp \left[\int_{0}^{n}\left(z_{i}^{*}-1\right) d \ln n\right]
$$

where

$$
z_{i}^{*} \equiv\left[\frac{\partial(n z)}{\partial n_{i}}\right]_{T, A, n_{j}}
$$

The exponential term is the fugacity coefficient when the molar area is the independent variable, analogous to the fugacity coefficient of a bulk gas when the independent variable is its molar volume. Equation (18) satisfies the Gibbs adsorption isotherm, Eq. (6).

Equation (18) is for the case when the equation of state is explicit in the spreading pressure. In the case when the equation of state is explicit in the molar area:

$$
f_{i}=\frac{\Pi A x_{i}}{B_{i \mathrm{~S}}} \exp \left[\int_{0}^{\Pi}\left(\bar{z}_{i}-1\right) d \ln \Pi\right]
$$

where

$$
\bar{z}_{i} \equiv\left[\frac{\partial(n z)}{\partial n_{i}}\right]_{T, \Pi, n_{j}}
$$


which is the partial molar compressibility of component $i$. Surface-phase activity coefficients are calculated from Eq. (5):

$$
\gamma_{i}=\frac{f_{i}}{x_{i} f_{i}^{\circ}}
$$

Equation (18) or (20) can be combined with a 2-d equation of state to determine the functionality of surfacephase activity coefficients. The $2-d$ virial equation is chosen to study the asymptotic behavior of $g^{\mathrm{ex}}$ at low coverage (Morrison and Ross, 1973):

$$
z=\frac{\Pi a}{R T}=1+\frac{B}{a}+\cdots=1+\frac{B \Pi}{R T}+\cdots
$$

Truncation after the 2-d second virial coefficient $B$ for pairwise interactions is justified if the surface coverage is sufficiently low for molecular clustering in triplets to be highly improbable. (The $2-d$ second virial coefficient $B$ is to be distinguished from the gas-solid second virial coefficient $B_{1 \mathrm{~S}}$ ). For a mixture:

$$
B_{m}=\sum_{i} \sum_{j} x_{i} x_{j} B_{i j}
$$

For a binary mixture $B_{m}=x_{1}^{2} B_{11}+2 x_{1} x_{2} B_{12}+x_{2}^{2} B_{22}$. For virial coefficient $B_{i j}$ (Hill and Greenschlag, 1961, Oh and Kim, 1974):

$$
B_{i j}=\int_{0}^{\infty}\left[1-e^{-U_{i j} / k T}\right] \pi r d r
$$

where $U_{i j}\{r\}$ is the interaction energy of molecules $i$ and $j$ as a function of their separation $r$.

Substitution of Eqs. (23) and (24) into (20) yields the fugacity of the $i$ th component of a mixture:

$$
f_{i}=\frac{\Pi A x_{i}}{B_{i \mathrm{~S}}} \exp \left[\frac{\left[\left(2 \sum_{j}\left(x_{j} B_{i j}\right)-B_{m}\right)\right] \Pi}{R T}\right]
$$

The standard-state fugacity for the pure adsorbate is:

$$
f_{i}^{\circ}=\frac{\Pi A}{B_{i s}} \exp \left[\frac{B_{i i} \Pi}{R T}\right]
$$

Substitution of (26) and (27) in (22) gives the activity coefficient of component 1 in a binary mixture of components 1 and 2:

$$
\gamma_{1}=\exp \left[\frac{x_{2}^{2} \delta_{12} \Pi}{R T}\right]
$$

using $\delta_{12} \equiv\left(2 B_{12}-B_{11}-B_{22}\right)$. Similarly for component no. 2:

$$
\gamma_{2}=\exp \left[\frac{x_{1}^{2} \delta_{12} \Pi}{R T}\right]
$$

Finally, substitution of Eqs. (28) and (29) into (3) gives an asymptotic expression for $g^{\text {ex }}$ :

$$
g^{\mathrm{ex}}=x_{1} x_{2} \delta_{12} \Pi
$$

Therefore from Eq. (12) the leading term for the excess area function is:

$$
a^{\mathrm{ex}}=x_{1} x_{2} \delta_{12}
$$

The excess area at the limit of zero coverage is finite and quadratic in composition. The value of $\delta_{12}$ is usually positive for bulk-gas mixtures; likewise positive deviations are the rule for adsorption on an energetically homogeneous surface. For adsorption on heterogeneous surfaces like zeolites and activated carbon, deviations from ideal mixing are negative (Valenzuela, Myers, Talu and Zwiebel, 1988) as shown below by experiment and by molecular simulation. The point here is not the sign but the functional form of the excess properties at the limit of zero coverage: both the excess Gibbs free energy and the excess area are quadratic in composition.

The excess area does not vanish for real adsorbates at the limit of zero amount adsorbed. This seems paradoxical at first sight since adsorbate-adsorbate interactions are negligible and the solution is ideal $\left(\Delta g^{\mathrm{ex}}=0\right)$. Equation (13) shows that the excess area at the limit of zero coverage has the form $(\infty-\infty)$. Thus there is no inconsistency in defining an ideal solution as one with zero $a^{\text {ex }}$, even though real adsorbed solutions have finite values of $a^{\text {ex }}$ at the limit of zero loading.

\subsection{Partial Exclusion of Large Molecules from Small Micropores}

The 2-d virial equation predicts that the excess properties are quadratic in composition at the limit of zero coverage but the equation diverges at high surface coverage. The model of partial exclusion of large molecules from small micropores (Dunne and Myers, 1994) is used to investigate the functionality of the excess properties at high surface coverage.

Molecules with effective diameters larger than the windows into microporous cavities cannot enter; the exclusion effect may be total or partial depending upon the pore-size distribution of the adsorbent. Consider adsorption of a binary mixture composed of large molecules (species no. 1) and small molecules (species no. 2) in a mixture of two adsorbents A (large, monodisperse pores) and B (small, monodisperse pores). Both molecules can enter the cavities of adsorbent $\mathrm{A}$ but 


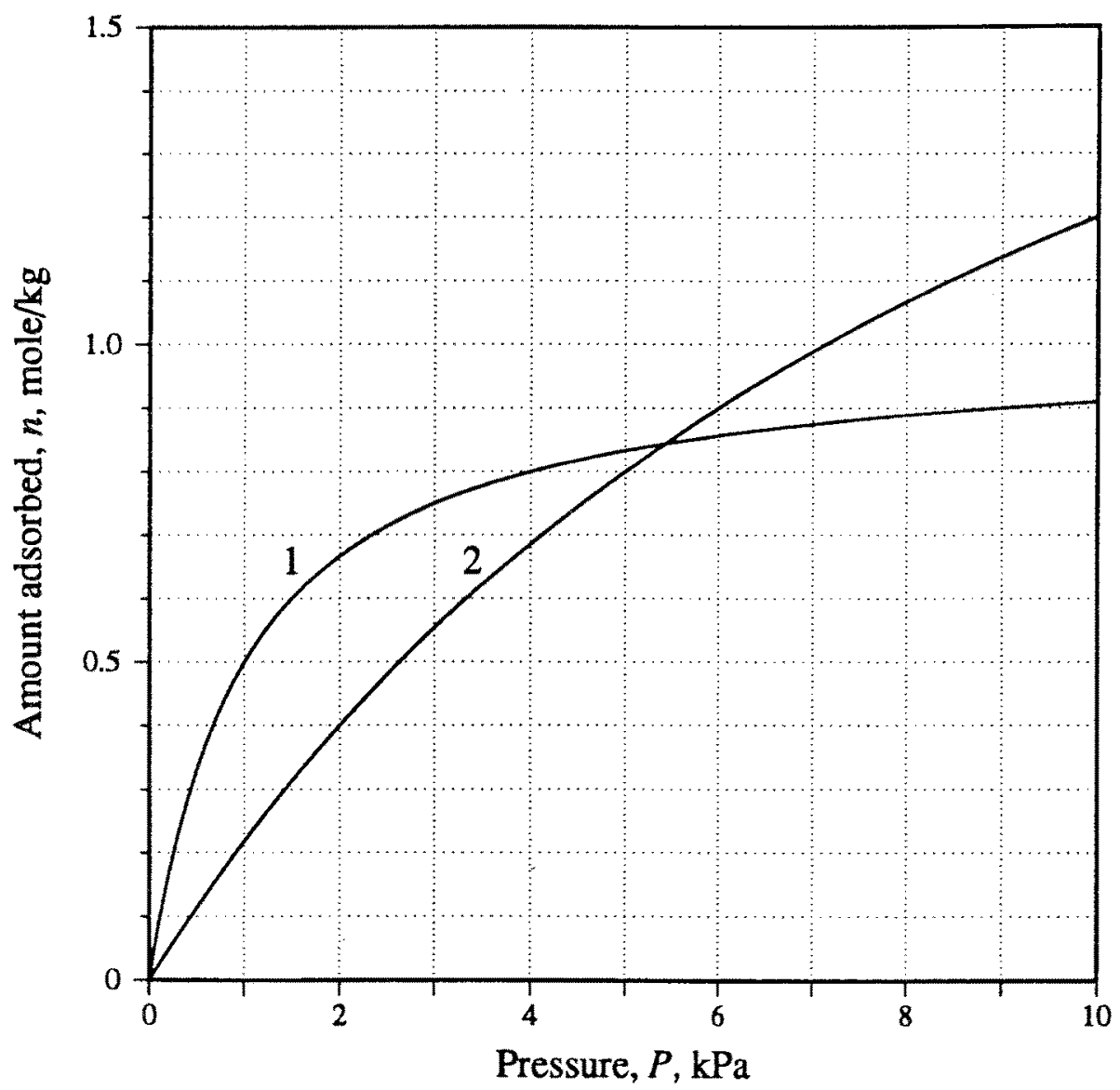

Fig. 1. Langmuirian adsorption isotherms. Component 1: $C=1, m=1$. Component 2: $C=0.1, m=1.488$.

molecules of species no. 1 are excluded from the micropores of adsorbent $B$.

The single-gas isotherms are:

$$
\begin{aligned}
& n_{1}=n_{1 \mathrm{~A}} \\
& n_{2}=n_{2 \mathrm{~A}}+n_{2 \mathrm{~B}}
\end{aligned}
$$

Mixtures compete for available adsorption space in adsorbent A but only the smaller molecule can adsorb in adsorbent $\mathrm{B}$.

Let the single-gas adsorption isotherms be represented by the Langmuir equation:

$$
n=\frac{m C f}{1+C f}
$$

where $m$ is the saturation capacity $(\mathrm{mol} / \mathrm{kg}), C$ is the affinity for the adsorbate, and $f$ is its gas-phase fugacity.

The Langmuir parameters are $m_{1 \mathrm{~A}}$ and $C_{1 \mathrm{~A}}$ for the large molecule in adsorbent $\mathrm{A}, m_{2 \mathrm{~A}}$ and $C_{2 \mathrm{~A}}$ for the small molecule in adsorbent $\mathrm{A}$, and $m_{2 \mathrm{~B}}$ and $C_{2 \mathrm{~B}}$ for the small molecule in adsorbent $B$.

Mixture VAE were generated for the partial exclusion model by adding competitive, ideal adsorption in adsorbent $\mathrm{A}$ to the non-competitive adsorption in adsorbent B. VAE data were analyzed using Eqs. (4)(13). The combined adsorbents were treated as a thermodynamic system, as if there were no information about the pore-size distribution of the adsorbent and the exclusion of the larger molecule from the smaller pores. This is different from the mixed-gas Langmuir model, which predicts ideal behavior. These calculations yield overall activity coefficients for the thermodynamic system of two adsorbents. Although competitive adsorption in adsorbent $A$ is ideal, the exclusion of molecule 1 from adsorbent $B$ generates non-ideal behavior for the combined adsorbents $\mathrm{A}$ and $\mathrm{B}$.

Although the functionality of the excess Gibbs free energy cannot be expressed in analytical form at high coverage for the theory of partial exclusion, an 


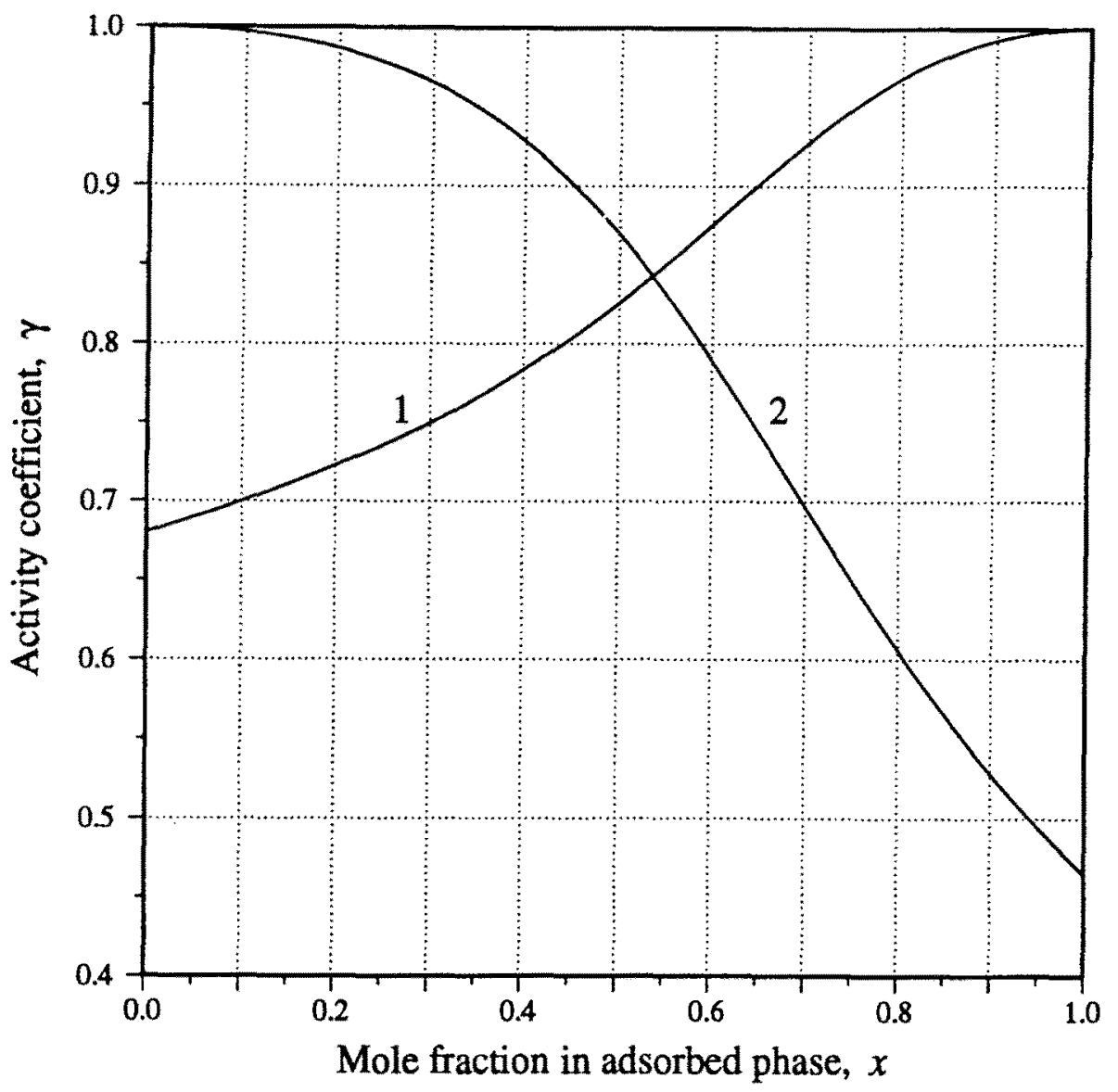

Fig. 2. Activity coefficients at high surface coverage $(P=10 \mathrm{~atm}$.) for partial exclusion of larger molecule (no. 1).

asymptotic expression for low coverage was derived:

$$
\frac{g^{\mathrm{ex}}}{R T}=-x_{1} x_{2}\left(\frac{\alpha \psi}{2 m_{1 \mathrm{~A}}}\right)
$$

where $\alpha=m_{2 \mathrm{~B}} /\left(m_{2 \mathrm{~A}}+m_{2 \mathrm{~B}}\right)$ is the fractional excluded volume and $\psi=\Pi A / R T$. From Eq. (12):

$$
\frac{a^{\mathrm{ex}}}{A}=-x_{1} x_{2}\left(\frac{\alpha}{2 m_{\mathrm{AA}}}\right)
$$

Thus in the absence of adsorbent $B$ which excludes the large molecules of species no. $1, \alpha=0$ and overall competitive adsorption is ideal. Exclusion causes negative deviations from ideal mixing.

Both the virial expansion, Eq. 30, and the partial exclusion model, Eq. 34, predict that the excess Gibbs free energy is quadratic in composition and linear in spreading pressure (and $\psi$ ) at the limit of zero coverage.

Since there is no analytical expression for $g^{\text {ex }}$ at high coverage, numerical calculations were performed for the following case: $m_{1 \mathrm{~A}}=1 \mathrm{~mol} / \mathrm{kg} ; m_{2 \mathrm{~A}}=1.488$ $\mathrm{mol} / \mathrm{kg} ; m_{2 \mathrm{~B}}=0.912 \mathrm{~mol} / \mathrm{kg} ; C_{1 \mathrm{~A}}=1.0 \mathrm{~atm}^{-1} ;$ $C_{2 \mathrm{~A}}=C_{2 \mathrm{~B}}=0.1 \mathrm{~atm}^{-1}$. The selectivity of adsorbent $A$ for component 1 relative to component 2 is $C_{1 \mathrm{~A}} / C_{2 \mathrm{~A}}=10$ and the fraction of excluded volume $\alpha=0.912 /(1.488+0.912)=0.38$. The single-gas isotherms for these parameters are plotted on Fig. 1 . Component 1 is preferentially adsorbed at low coverage but the smaller component 2 is preferentially adsorbed at high coverage. This type of behavior is typical for different-sized molecules of a homologous series of compounds.

Activity coefficients at high coverage are shown for a constant pressure of $10 \mathrm{~atm}$. on Fig. 2 and for constant $\psi=2.22 \mathrm{~mol} / \mathrm{kg}$ on Fig. 3. The curves on Figs. 2 and 3 coincide at $x_{1}=0.5$, where $P=10 \mathrm{~atm}$ and $\psi=2.22 \mathrm{~mol} / \mathrm{kg}$. The curves at constant $\psi$ in Fig. 3 are more symmetric in composition than the curves at constant $P$ in Fig. 2. 


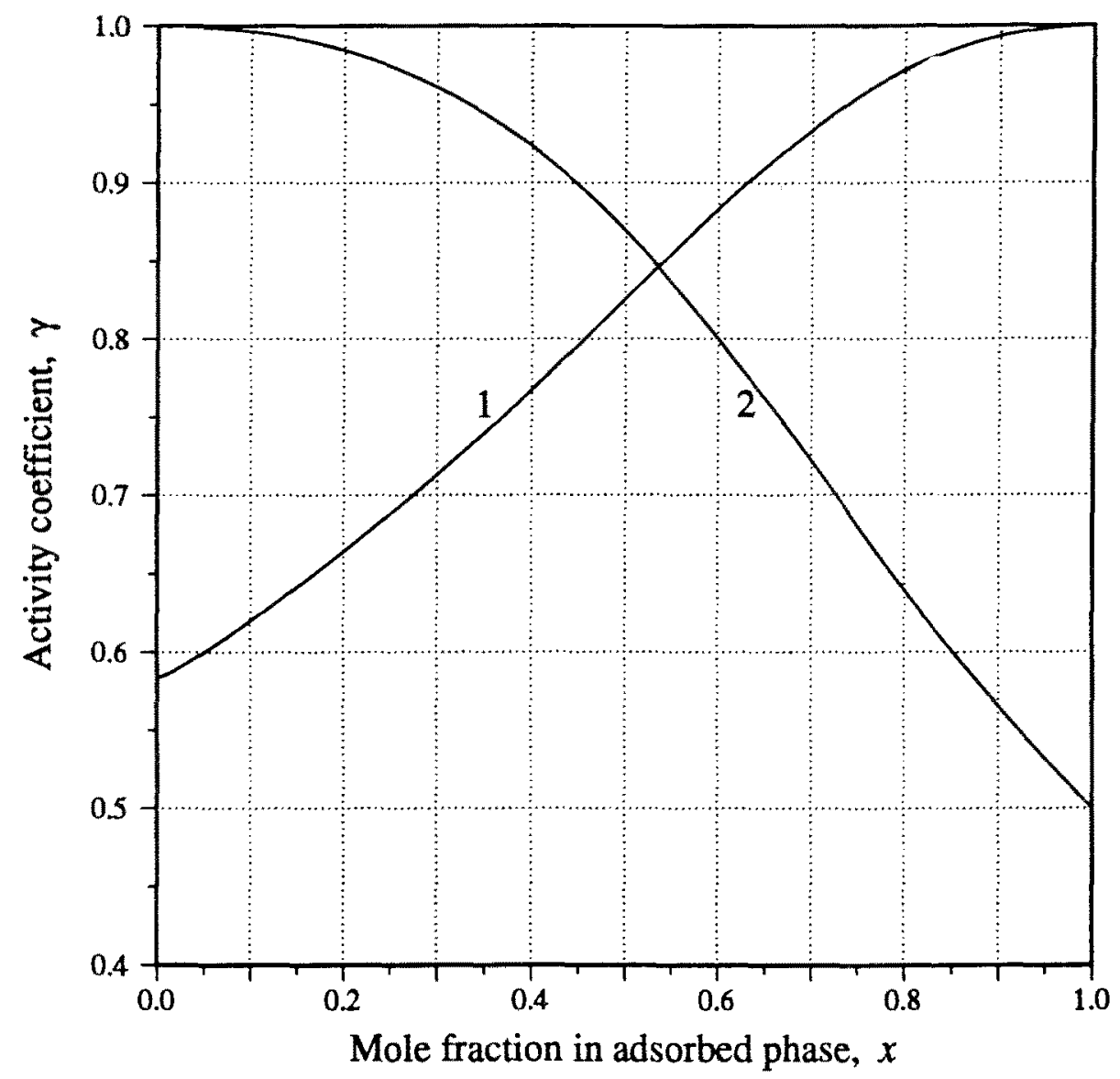

Fig. 3. Activity coefficient at high surface coverage $(\psi=2.22 \mathrm{~mol} / \mathrm{kg}$ ) for partial exclusion of larger molecule (no. 1). These curves coincide with those on Fig. 2 at $x_{1}=0.5$.

The Gibbs-Duhem differential consistency test derived from Eq. (3) for activity coefficients at constant $\psi$ is:

$$
\frac{\partial \ln \gamma_{1}}{\partial x_{1}}=-\frac{\partial \ln \gamma_{2}}{\partial x_{1}}
$$

at the equimolar composition. The constant- $\psi$ curves on Fig. 3 obey Eq. (36):

$$
\begin{aligned}
& \left(\partial \ln \gamma_{1} / \partial x_{1}\right)=+0.713 \\
& \left(\partial \ln \gamma_{2} / \partial x_{1}\right)=-0.713
\end{aligned}
$$

The constant- $P$ curves on Fig. 2 fail to obey Eq. (36):

$$
\begin{aligned}
& \left(\partial \ln \gamma_{1} / \partial x_{1}\right)=+0.566 \\
& \left(\partial \ln \gamma_{2} / \partial x_{1}\right)=-0.802
\end{aligned}
$$

Experimental data for adsorption of mixtures are measured at constant $P$, not at constant $\psi$. Activity coefficients derived from isobaric data cannot be fit by any of the equations used for VLE (Wilson, Margules, etc.), regardless of the number of parameters. (Talu and Zwiebel, 1986) VLE data obey Eq. (36); isobaric VAE data do not obey Eq. (36).

The constant- $\psi$ activity coefficients in Fig. 3 are reproduced with an average error of $2 \%$ by the quadratic expression $\left(g^{\mathrm{ex}} / R T\right)=C x_{1} x_{2}, \ln \gamma_{1}=C x_{2}^{2}, \ln \gamma_{2}=$ $C x_{1}^{2}$, with $C=-0.66$ at $\psi=2.22 \mathrm{~mol} / \mathrm{kg}$.

The excess Gibbs free energy at constant $\psi$ is exactly quadratic in composition at the limit of zero coverage and approximately quadratic in composition at high coverage.

The excess Gibbs free energy function varies with $\psi$. The limiting slope at $\psi=0$ is given by Eq. (34):

$$
\frac{\partial\left[g^{\mathrm{ex}} /\left(R T x_{1} x_{2}\right)\right]}{\partial \psi}=-\frac{\alpha}{2 m_{1 \mathrm{~A}}}=-\frac{0.38}{2(1.0)}=-0.19
$$

The isothermal variation of adsorbed-phase activity coefficients with composition and spreading pressure is 


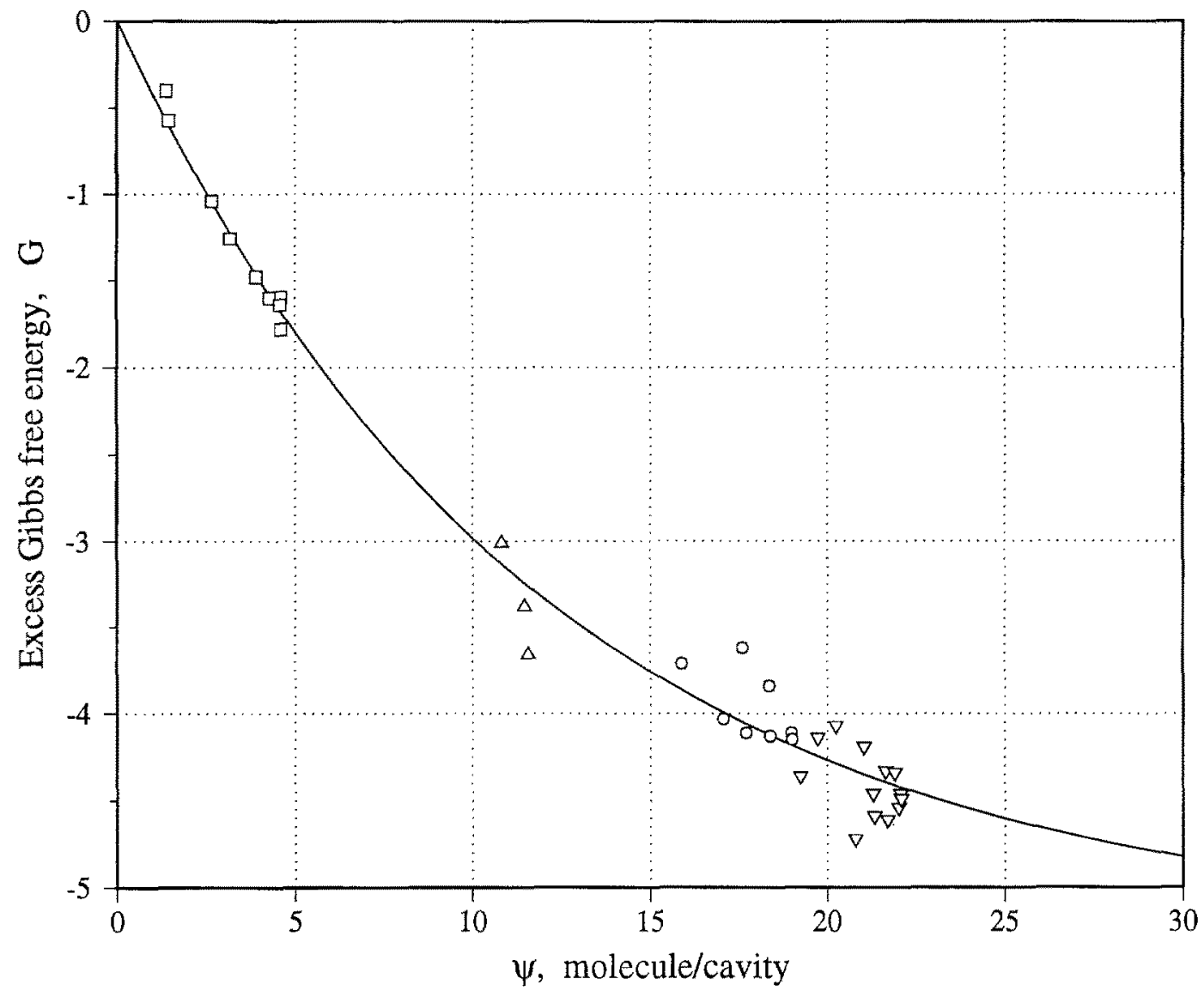

Fig. 4. Excess Gibbs free energy function $G=g^{\mathrm{ex}} / R T x_{1} x_{2}$ versus $\psi$ from molecular simulation of mixtures $i-\mathrm{C}_{4} \mathrm{H}_{10}$ and $\mathrm{C}_{2} \mathrm{H}_{4}$ in zeolite $13 \mathrm{X}$ at $298.15 \mathrm{~K}$. (口): $P=1 \mathrm{kPa} ;(\Delta): P=10 \mathrm{kPa} ;(0): P=70 \mathrm{kPa} ;(\nabla): P=137 \mathrm{kPa}$. Solid line: Eq. $(38)$ with $C=-5.24, \beta=0.084$.

given approximately by (Valenzuela and Myers, 1989):

$$
\frac{g^{\mathrm{ex}}}{R T}=x_{1} x_{2} C\left(1-e^{-\beta \psi}\right)
$$

For the above example, two constants $C=0.5 \mathrm{~kg} / \mathrm{mol}$ and $\beta=-0.38(\mathrm{~kg} / \mathrm{mol})$ reproduce the activity coefficients with a average error of $2 \%$ in the range $0<\psi<3 \mathrm{~mol} / \mathrm{kg}$, which corresponds to $0<P<20$.

Activity coefficients are required to calculate the composition of the adsorbed phase; the total amount adsorbed is obtained from Eq. (13) and the excess area. From Eq̨s. (12) and (38):

$$
\frac{a^{\mathrm{ex}}}{A}=x_{1} x_{2} \beta C e^{-\beta \psi}
$$

\section{Molecular Simulation}

Monte Carlo simulations of adsorption of binary mixtures of isobutane and ethylene on zeolite $13 \mathrm{X}$ at $298.15 \mathrm{~K}$ were performed (Karavias and Myers, 1991). The Lennard-Jones $12-6$ potential was used for gas-gas dispersion/repulsion interactions, and the Lennard-Jones Devonshire theory for gas-solid dispersion/repulsion interactions. Isobutane was assumed to be nonpolar and the ethylene molecule had a point quadrupole moment of $3.92 \times 10^{-26}$ esu$\mathrm{cm}^{2}$ placed at its center. The electric field strength inside the $13 \mathrm{X}$ cavity was calculated from partial charges of 0.58 placed on the sodium ions. The ion-quadrupole interaction between the sodium ions of the zeolite and the quadrupole moment of ethylene generated electrostatic energy terms, and the interaction of the electric field with the polarizable adsorbate molecules generated induced electrostatic energy terms. Because of the approximate nature of the molecular model, comparison of the simulation with the experimental single-gas adsorption isotherms showed systematic errors of $10 \%$ for isobutane and $25 \%$ for ethylene. 


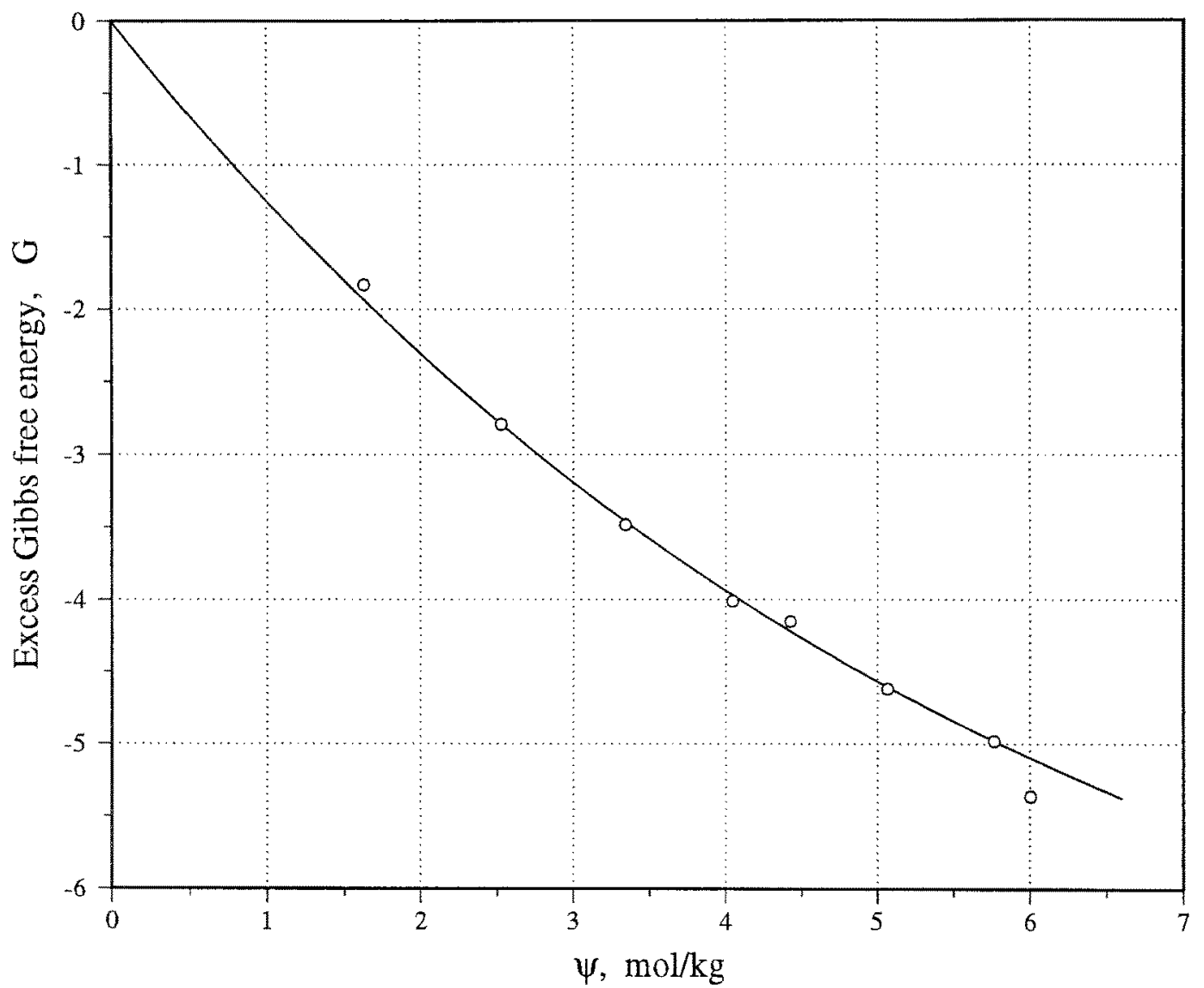

Fig. 5. Excess Gibbs free energy function $G=g^{\mathrm{cx}} / R T x_{1} x_{2}$ versus $\psi$ from experimental data (Talu and Zwiebel, 1986) on adsorption of $\mathrm{C}_{3} \mathrm{H}_{8}$ and $\mathrm{H}_{2} \mathrm{~S}$ on $H$-mordenite at $303.15 \mathrm{~K}$. (o): Experimental points. Solid line: Eq. (38) with $C=-7.92, \beta=0.172$.

Activity coefficients were calculated from the simulation results for binary adsorption of isobutane and ethylene. Figure 4 shows that Eq. (38) fits the data with an average absolute error of $4 \%$. Some of the scatter in the data at high surface coverage is due to the weak dependence of $g^{\mathrm{ex}} / R T x_{1} x_{2}$ on composition. Activity coefficients at infinite dilution at high coverage are as small as $\gamma^{\infty}=0.2$. This highly nonideal behavior is due primarily to the difference in polarities of isobutane and ethylene molecules in the strong electric field inside $13 \mathrm{X}$ cavity.

\section{Experiment}

Activity coefficients were measured by (Talu and Zwiebel, 1986) for binary mixtures of propane and hydrogen sulfide on $\mathrm{H}$-mordenite at $30^{\circ} \mathrm{C}$. Excess Gibbs free energy as a function of $\psi$ is shown on Fig. 5. The solid line is the fit of Eq. (38), which agrees with the data within an average absolute error of $2 \%$. This is a highly nonideal solution, with activity coefficients at infinite dilution $\gamma^{\infty}<0.1$. Data contain both constant $P$ and $Y$ planes. As in the case of the molecular simulations, the scatter in the data at high surface coverage is partly ascribed to the weak dependence of $g^{\mathrm{ex}} / R T x_{1} x_{2}$ on composition.

\section{Conclusions}

Theory, molecular simulation, and experiment all show that the two-parameter Eq. (38) fits the excess free energy function over the entire range of surface coverage with less than $5 \%$ error, which is sufficiently accurate for most engineering calculations. Two parameters provide a complete description of isothermal, binary adsorption equilibrium, from low to high coverage. 
The quadratic dependence of the excess Gibbs free energy function on the composition of the adsorbed phase is exact at the limit of zero coverage, and approximate at high coverage.

\section{References}

Dunne, J. and A.L. Myers, "Adsorption of gas mixtures in micropores: effect of difference in size of adsorbate molecules," Chem. Eng. Sci, in press (1994).

Hill, TL. and S. Greenschlag, "Statistical mechanics of monatomic systems in an external periodic potential field: 1 . Introduction, virial expansion, and classical second virial coefficient," $J$. Chem. Phys, 34, 1538 (1961).

Hoory, S.E. and J.M. Prausnitz, "Monolayer adsorption of gas mixtures on homogeneous and heterogeneous solids," Chem. Eng. Sci., 22, 1025 (1967).

Karavias, F. and A.L. Myers, "Monte Carlo simulation of binary gas adsorption in zeolite cavities," Molecular Simulation, 8, 51-72 (1991).

Morrison, I.D. and S. Ross, "The second and third virial coefficients of a two-dimensional gas," Surf. Sci, 39, 21 (1973).

Myers, A.L. and J.M. Prausnitz, "Thermodynamics of mixed-gas adsorption." AlChE J., 11, 121 (1965).
Oh, B.K. and S.K. Kim, "Virial expansion of the second layer in physical adsorption, an $a b$ initio calculation for helium on argon crystal," J. Chem. Phys., 61, 1797 (1974).

Prausnitz, J.M, R.N. Lichtenthaler, and E.G. Azevedo, Molecular Thermodynamics of Fluid-Phase Equilibria, Prentice-Hall, Engelwood Cliffs, N.J. 1986.

Smith, J.M. and H.C. Van Ness, Introduction to Chemical Engineering Thermodynamics, Third Ed., McGraw-Hill, N.Y., p. 331, 1975.

Steele, W.A., The Interaction of Gases with Solid Surfaces, Pergamon Press, Glasgow, 1974.

Talu, O. and A.L. Myers, "Rigorous thermodynamic treatment of gas adsorption," AIChE J., 34; 1887 (1988).

Talu, $\mathrm{O}$. and I. Zwiebel, "Multicomponent adsorption equilibria of nonideal mixtures," AIChE J, 32, 1263 (1986).

Valenzuela, D.P. and A.L. Myers, Adsorption Equilibrium Data Handbook, Prentice-Hall, Engelwood Cliffs, N.J., p. 216, 1989.

Valenzuela, D.P., A.L. Myers, O. Talu, and I. Zwiebel, "Adsorption of gas mixtures: effect of energetic heterogeneity," AIChE J., 34, 397 (1988).

Van Ness, H.C., "Adsorption of gases on solids," I. \& E.C. Fundamentals, 8, 464, 1969.

Van Ness, H.C. and M.M. Abbott, Classical Thermodynamics of Non-electrolyte Solutions, McGraw-Hill, N.Y., p. 289, 1982. 